\title{
Humoral immune response after different SARS-CoV-2 vaccination regimens
}

\author{
Ruben Rose ${ }^{1 \dagger}$, Franziska Neumann $^{2 \dagger}$, Olaf Grobe ${ }^{2}$, Thomas Lorentz ${ }^{2}$, Helmut Fickenscher ${ }^{1}$ and
} Andi Krumbholz ${ }^{1,2^{*}}$ (i)

\begin{abstract}
Background: The humoral immune response after primary immunisation with a SARS-CoV-2 vector vaccine (AstraZeneca AZD1222, ChAdOx1 nCoV-19, Vaxzevria) followed by an mRNA vaccine boost (Pfizer/BioNTech, BNT162b2; Moderna, m-1273) was examined and compared with the antibody response after homologous vaccination schemes (AZD1222/AZD1222 or BNT162b2/BNT162b2).
\end{abstract}

Methods: Sera from 59 vaccinees were tested for anti-SARS-CoV-2 immunoglobulin G (lgG) and virus-neutralising antibodies (VNA) with three IgG assays based on (parts of) the SARS-CoV-2 spike (S)-protein as antigen, an IgG immunoblot (additionally contains the SARS-CoV-2 nucleoprotein (NP) as an antigen), a surrogate neutralisation test (sVNT), and a Vero-cell-based virus-neutralisation test (cVNT) with the B.1.1.7 variant of concern (VOC; alpha) as antigen. Investigation was done before and after heterologous ( $n=30$ and 42) or homologous booster vaccination (AZD1222/AZD1222, $n=8 / 9 ;$ BNT162b2/BNT162b2, $n=8 / 8$ ). After the second immunisation, a subgroup of 26 ageand gender-matched sera (AZD1222/mRNA, $n=9$; AZD1222/AZD1222, $n=9$; BNT162b2/BNT162b2, $n=8$ ) was also tested for VNA against VOC B.1.617.2 (delta) in the cVNT. The strength of IgG binding to separate SARS-CoV-2 antigens was measured by avidity.

Results: After the first vaccination, the prevalence of lgG directed against the (trimeric) SARS-CoV-2 S-protein and its receptor binding domain (RBD) varied from 55-95\% (AZD1222) to 100\% (BNT162b2), depending on the vaccine regimen and the SARS-CoV-2 antigen used. The booster vaccination resulted in 100\% seroconversion and the occurrence of highly avid $\operatorname{lgG}$, which is directed against the S-protein subunit 1 and the RBD, as well as VNA against VOC B.1.1.7, while anti-NP IgGs were not detected. The results of the three anti-SARS-CoV-2 IgG tests showed an excellent correlation to the VNA titres against this VOC. The agreement of CVNT and sVNT results was good. However, the sVNT seems to overestimate non- and weak B.1.1.7-neutralising titres. The anti-SARS-CoV-2 IgG concentrations and the B.1.1.7-neutralising titres were significantly higher after heterologous vaccination compared to the homologous AZD1222 scheme. If VOC B.1.617.2 was used as antigen, significantly lower VNA titres were measured in the cVNT, and three (33.3\%) vector vaccine recipients had a VNA titre < 1:10.

\footnotetext{
* Correspondence: krumbholz@infmed.uni-kiel.de

${ }^{\dagger}$ Ruben Rose and Franziska Neumann contributed equally to this work. 'Institut für Infektionsmedizin, Christian-Albrechts-Universität zu Kiel und Universitätsklinikum Schleswig Holstein, Campus Kiel, Brunswiker Straße 4, D-24105 Kiel, Germany

2Labor Dr. Krause und Kollegen MVZ GmbH, Steenbeker Weg 23, D-24106 Kiel, Germany
}

(c) The Author(s). 2022 Open Access This article is licensed under a Creative Commons Attribution 4.0 International License, which permits use, sharing, adaptation, distribution and reproduction in any medium or format, as long as you give appropriate credit to the original author(s) and the source, provide a link to the Creative Commons licence, and indicate if changes were made. The images or other third party material in this article are included in the article's Creative Commons licence, unless indicated otherwise in a credit line to the material. If material is not included in the article's Creative Commons licence and your intended use is not permitted by statutory regulation or exceeds the permitted use, you will need to obtain permission directly from the copyright holder. To view a copy of this licence, visit http://creativecommons.org/licenses/by/4.0/ The Creative Commons Public Domain Dedication waiver (http://creativecommons.org/publicdomain/zero/1.0/) applies to the data made available in this article, unless otherwise stated in a credit line to the data. 
Conclusions: Heterologous SARS-CoV-2 vaccination leads to a strong antibody response with anti-SARS-CoV-2 IgG concentrations and VNA titres at a level comparable to that of a homologous BNT162b2 vaccination scheme. Irrespective of the chosen immunisation regime, highly avid IgG antibodies can be detected just 2 weeks after the second vaccine dose indicating the development of a robust humoral immunity. The reduction in the VNA titre against VOC B.1.617.2 observed in the subgroup of 26 individuals is remarkable and confirms the immune escape of the delta variant.

Keywords: COVID-19, Vaccination schemes, Immunoglobulin G, Maturity process, Virus variants of concern, Virus neutralisation

\section{Background}

Since spring 2020, the pandemic caused by the severe acute respiratory syndrome coronavirus 2 (SARS-CoV-2) [1] is ongoing and represents a global challenge. The availability of safe and effective vaccinations is seen as one of the most important pillars in containing the pandemic $[2,3]$. Within a few months, intensive research activities led to the development of several highly effective SARS-CoV-2 vaccines [3-5]. In addition to the induction of cellular immunity, their administration should stimulate the formation of virus-neutralising antibodies (VNA) that bind to epitopes of the viral spike (S)-protein and its receptor binding domain (RBD) and, thus, prevent cell entry $[3,6,7]$.

Four SARS-CoV-2 vaccines have received conditional approval in the European Union. These vaccines are based on two different technologies [8]. For the messenger ribonucleic acid (mRNA) vaccines from Pfizer/BioNTech (BNT162b2) and Moderna (mRNA-1273), the genetic information for the S-protein was optimised and the mRNA was packaged in liposomes. After inoculation, the muscle cells directly expressed this stable and highly immunogenic viral surface protein $[2,6]$. In vector vaccines, replication-deficient human (Ad26.COV2; Janssen) or chimpanzee adenoviruses (ChAdOx1 nCoV19/AZD1222, Vaxzevria; AstraZeneca, hereinafter referred to as AZD1222) are used to introduce the genetic information of the SARS-CoV-2 S-protein into the cells, followed by transcription of deoxyribonucleic acid into mRNA and expression of the S-protein $[2,6]$.

Due to the widespread use of these vaccines, rare and sometimes unexpected side effects have been reported. Particularly noteworthy are cases of immune thrombotic thrombocytopenia, which predominantly occurred in women under 50 years of age within 1 month after the initial vaccination with AZD1222 [5]. Many of these patients developed cerebral sinus venous thrombosis or splanchnic vein thrombosis and presented antibodies to platelet factor 4 but without previous exposure to heparin [5]. Due to this rare but serious side effect, AZD1222 is no longer unreservedly recommended by the Standing Vaccination Commission (STIKO) of the Robert Koch Institute for individuals under 60 years of age. The STIKO suggests that a vaccination with AZD1222 that has already started should be completed with an mRNA vaccine $[9,10]$. Due to the sharp increase in the delta variant of concern (VOC; Pango-lineage [11] B.1.617.2) in Germany, the STIKO has revised its recommendations once more. Since July 1, 2021, all AZD1222 first vaccinated persons have been recommended to complete the second vaccination with an mRNA vaccine [12]. Animal experiments indicated very good humoral and cellular immunity after heterologous vaccination $[13,14]$. There is, however, so far limited knowledge on the benefit of the heterologous vaccination scheme in humans. First results indicated a higher prevalence of short-lived side effects following the heterologous boost dose compared to the homologous counterpart [15]. Meanwhile, a few studies have been published for the immunogenicity of the AZD1222/mRNA vaccine regimen [16-22].

In this report, we compare the SARS-CoV-2-specific immunoglobulin G (IgG) response after heterologous immunisation with that elicited by homologous vaccination schedules. We also focus on the developing antiSARS-CoV-2 IgG avidity as a parameter for IgG maturity and binding strength. Finally, we investigate the development of VNA against two prevalent VOCs. The various methods described are being used to thoroughly study vaccine-induced humoral immune response magnitude and surrogate efficacy. We believe that the results obtained in this study will help to better understand the effects and possibly benefits of a heterologous vaccination regimen.

\section{Methods}

The anti-S and anti-RBD IgG response after heterologous immunisation with a SARS-CoV-2 vector vaccine as prime and an mRNA vaccine as boost was compared to that after homologous vaccination with vector or mRNA vaccines. This setting also includes monitoring of IgG avidity and of virus-neutralising capacities. Fortyseven female and twelve male vaccinees with a median age of 31 years (age span 18-61 years) were recruited for this study and gave their informed consent. The prior SARS-CoV-2 infection status was not queried. Since it 
was not clear in advance how many individuals could be recruited for the study, we did not estimate the required number of cases. Rather, all vaccinees who declared their willingness to participate were included. Forty-two of them received a heterologous immunisation scheme $(N$ = 40, AZD1222/BNT162b2; $N=2$, AZD1222/mRNA1273), while nine and eight vaccinees received a homologous scheme of the vector vaccine AZD1222 or the mRNA vaccine BNT162b2, respectively. The first blood sample was taken immediately before the second vaccination and the second about 2 weeks later (median 1416 days, time span 10-34 days, Table 1). Several individuals only contributed samples before or after vaccine boost and do not have matched data. The ethics committee of the medical faculty of the Christian-AlbrechtsUniversität zu Kiel (Kiel, Germany) approved the study design (D467/20, 16.04.2020, amendment 02.02.2021). We examined the early humoral immune response (other samples obtained a few days to weeks after the initial immunisation with AZD1222) of most of the subjects in a previous study. In addition, sera obtained from three vaccinees after the initial immunisation with BNT162b2 $(N=2)$ and AZD1222 $(N=1)$ have already been tested in frame of the previous study [23]. In this respect, we consider it justified to include these individuals (and few sera) in the present report and to demonstrate the results before and after the second vaccination.

\section{Anti-SARS-CoV-2-specific IgG immunoassays}

The sera were tested with the SERION ELISA agile SARS-COV-2 IgG assay (S-protein as antigen; Institut
Virion\Serion GmbH, Würzburg, Germany; negative: < $10 \mathrm{U} / \mathrm{ml}$, borderline range $10-14 \mathrm{U} / \mathrm{ml}$, positive $\geq 15 \mathrm{U} /$ $\mathrm{ml}$; linearity range $3-250 \mathrm{U} / \mathrm{ml}$ ) and the Abbott SARSCoV-2 IgG II Quant assay (RBD as antigen; Abbott, Wiesbaden, Germany; cut-off $=50 \mathrm{AU} / \mathrm{ml}$ ) as described previously [23]. In addition, the LIAISON ${ }^{\circ}$ SARS-CoV-2 Trimeric S IgG assay (cut-off $=33.8$ Binding Antibody Units $(\mathrm{BAU}) / \mathrm{ml}$; linearity range 4.81 to $2080 \mathrm{BAU} / \mathrm{ml}$ ) was included as a further assay on a LIAISON ${ }^{\circ} \mathrm{XL}$ system (both Diasorin S.p.A, Saluggia, Italy). According to the manufacturer, this quantitative chemiluminescence immunoassay detects IgG directed against the trimeric S-protein and has an excellent clinical sensitivity and specificity of $98.7 \%$ and $99.5 \%$, respectively. The high diagnostic value of this test has also been demonstrated in a recent seroprevalence study [24]. The results of the three IgG assays were given in BAU per $\mathrm{ml}$, using the manufacturer's conversion factors, which were based on measurements of the WHO International Standard Anti-SARS-CoV-2 Immunoglobulin (NIBSC code 20136) [25]. As in our previous studies, we rate the borderline test results of the SERION ELISA agile SARS-COV2 IgG assay as positive [23, 26]. If a serum had an antiSARS-CoV-2 IgG concentration above the linearity range, this sample was 1:10 (SERION ELISA agile SARSCOV-2 IgG assay) or 1:20 (LIAISON ${ }^{\circ}$ SARS-CoV-2 Trimeric $S$ IgG assay) diluted in the manufacturer's dilution buffer and then measured again. The concentration was then recalculated. Since the linearity of the assay is no longer given after dilution, the measured values determined in this way may not entirely correspond to reality. We consider this effect to be negligible.

Table 1 Individuals included in this study

\begin{tabular}{|c|c|c|c|c|c|c|}
\hline Study groups & $\begin{array}{l}\text { Number of } \\
\text { individuals after 1st/ } \\
\text { 2nd vaccination }\end{array}$ & $\begin{array}{l}\text { Median } \\
\text { age in } \\
\text { years }\end{array}$ & $\begin{array}{l}\text { Age or age } \\
\text { span in } \\
\text { years }\end{array}$ & $\begin{array}{l}\text { Gender } \\
\text { (female/ } \\
\text { male) }\end{array}$ & $\begin{array}{l}\text { Time (median) from } 1 \mathrm{st} \\
\text { vaccination up to } 1 \text { st serum } \\
\text { sampling in days }\end{array}$ & $\begin{array}{l}\text { Time (median) from } 2 \text { nd } \\
\text { vaccination up to } 2 \text { nd serum } \\
\text { sampling in days }\end{array}$ \\
\hline \multicolumn{7}{|l|}{$\begin{array}{l}\text { Heterologous } \\
\text { vaccination } \\
\text { scheme }\end{array}$} \\
\hline $\begin{array}{l}\text { AZD1222/ } \\
\text { BNT162b2 }\end{array}$ & $28 / 40$ & 27 & $18-56$ & $32 / 8$ & 69 & 15 \\
\hline $\begin{array}{l}\text { AZD1222/ } \\
\text { BNT162b2 } \\
\text { (subgroup) }+\end{array}$ & $-/ 9$ & 43 & $23-56$ & $7 / 2$ & Not applicable & 14 \\
\hline $\begin{array}{l}\text { AZD1222/ } \\
\text { mRNA-1273 }\end{array}$ & $2 / 2$ & $\neq$ & 24,45 & $2 / 0$ & $64,69(\ddagger)$ & $10,14(\ddagger)$ \\
\hline \multicolumn{7}{|l|}{$\begin{array}{l}\text { Homologous } \\
\text { vaccination } \\
\text { scheme }\end{array}$} \\
\hline AZD1222 & $8 / 9$ & 41 & $23-61$ & $6 / 3$ & 69 & 16 \\
\hline BNT162b2 & $8 / 8$ & 35 & $23-51$ & $7 / 1$ & 34 & 14 \\
\hline
\end{tabular}

†After the 2nd vaccination, several sera were also tested for the presence of virus-neutralising antibodies against the SARS-CoV-2 delta variant of concern (B.1.617.2). For this purpose, individuals from the group of heterologous vaccinations whose age and gender largely corresponded to those with homologous vaccinations were chosen. This subgroup is separately presented. $\neq$ Calculating the median does not make sense if there are two values 


\section{Anti-SARS-CoV-2 IgG immunoblots including measurement of IgG avidities}

The sera were tested in the recomLine SARS-CoV-2 IgG assay using the Dynablot Plus system together with a BLOTrix reader and the recomScan software (all from Mikrogen $\mathrm{GmbH}$, Neuried, Germany) as reported previously $[23,26]$. This immunoblot consists of a nitrocellulose strip on which the recombinant SARS-CoV-2 nucleoprotein (NP) as well as the S1-and RBD-subunits of its S-protein are separately spotted. In addition, the blot carries the recombinant NPs of four seasonal human coronaviruses (HCoVs 229E, NL63, OC43, and HKU1). By comparing the IgG binding to the SARS$\mathrm{CoV}-2$ antigens in the presence and absence of the avidity reagent, the binding strength was automatically determined and assessed $[23,26]$. The results were used to assign IgG avidity to four categories: no avidity detectable $(=0)$, low avidity $(=1)$, intermediate avidity $(=2)$ and high $(=3)$ avidity [23].

\section{Measurement of SARS-CoV-2-neutralising antibodies}

The sera were examined for their virus-neutralising capacities. First, a surrogate assay was used by strictly following the manufacturer's instructions (TECO ${ }^{\circ}$ SARSCoV-2 Neutralisation Antibody ELISA; TECOmedical AG, Sissach, Switzerland). In this competitive assay, the human angiotensin-converting enzyme 2 (ACE-2) was attached to the solid phase while peroxidase-conjugated RBD was present in the liquid phase. If the human serum contained RBD-specific antibodies, binding of RBD to ACE-2 was prevented. Hence, after washing steps, the colour reaction turned out to be weaker compared to a RBD-antibody-free serum sample. According to the manufacturer, it is assumed from an inhibition of RBD to ACE-2 binding $\geq 20 \%$ that VNA are present [23].

Second, dilutions of each serum were tested in triplicate in an in-house 96-well format Vero-cell-based neutralisation assay (cVNT) as previously reported [23]. In brief, $2.5 \times 10^{4}$ Vero cells (order no. 605372, CLS Cell Lines Service $\mathrm{GmbH}$, Eppelheim, Germany) were seeded per well and incubated at $37^{\circ} \mathrm{C}$ under standard conditions. On the next day, sera were heat-inactivated $\left(56^{\circ} \mathrm{C}\right.$ for $30 \mathrm{~min})$ and diluted in a cell culture medium (1:10, 1 : 20, 1:40, 1:80, 1:160, 1:320, 1:640, and 1:1280). The latter consists of Dulbecco's modified Eagle's medium supplemented with $3.7 \mathrm{~g} / \mathrm{l} \mathrm{NaHCO} 3,4.5 \mathrm{~g} / \mathrm{l}$ glucose, $2 \mathrm{mM} \mathrm{L-}$ glutamine, and $1 \%(\mathrm{v} / \mathrm{v})$ of Pen-Strep-Fungi mix containing $10,000 \mathrm{U} / \mathrm{ml}$ penicillin, $10 \mathrm{mg} / \mathrm{ml}$ streptomycin, and $25 \mu \mathrm{g} / \mathrm{ml}$ amphotericin B (all reagents from Bio\&SELL $\mathrm{GmbH}$, Feucht, Germany). Then, $25 \mu \mathrm{l}$ of the serum dilution was mixed with $25 \mu$ of virus suspension containing 50 plaque-forming units (pfu) of either an own VOC B.1.1.7 strain (alpha, from January 2021) or an own VOC B.1.617.2 strain (delta, from June 2021; both viruses were obtained after cultivation of material obtained from the upper respiratory tract of SARS-CoV-2 patients in Vero cells as described previously [27]). The resulting $50 \mu \mathrm{l}$ was incubated for $1 \mathrm{~h}$ at $37^{\circ} \mathrm{C}$. Meanwhile, Vero cells were washed with phosphate-buffered saline (PBS, Bio\&SELL). Then, $50 \mu \mathrm{l}$ of the virus-serum dilutions was pipetted on the prepared cells followed by $1 \mathrm{~h}$ of incubation on a shaker at room temperature. Thereafter, $50 \mu \mathrm{l}$ of a fresh cell culture medium supplemented with $20 \%$ foetal calf serum $(\mathrm{v} / \mathrm{v})$ was added per well and plates were incubated for 4 days under standard conditions. Then, cells were fixed by addition of $4 \%(\mathrm{w} /$ v) paraformaldehyde in PBS and stained with an aqueous solution of $0.1 \%(\mathrm{w} / \mathrm{v})$ crystal violet and $20 \%(\mathrm{v} / \mathrm{v})$ methanol. The cytopathic effect (CPE) formation was compared with an untreated cell control (medium only) and a viral control (50 pfu). A serum dilution $>1: 10$ (titre) that prevented CPE formation in at least two of three wells compared with the viral control was valued as containing neutralising antibodies. Recent data indicate that titres from around 1:100 are associated with a high vaccine effectiveness of $>80 \%$ [28]. When an exact titre could not be provided by the eye, the geometric mean of the two adjacent titres was calculated $[23,26]$.

\section{Data evaluation and statistical calculations}

Data were statistically analysed by the help of the GraphPad Prism version 9.1.2 software (GraphPad Software, San Diego, CA, USA). In most cases, the Kruskal-Wallis test, an adjusted, non-paired and non-parametric test, was applied. The Wilcoxon test, a paired non-parametric test, was chosen to compare the median VNA titre differences against VOC B.1.1.7 and VOC B.1.617.2. To compare the frequencies of the measured anti-SARSCoV-2 IgG avidity indices as a function of the vaccination scheme used, Fisher's exact test was applied, adjusted by the Bonferroni correction for multiple testing. The level of significance was generally set at $P=0.05$. Furthermore, we calculated the Spearman correlation coefficient to demonstrate the correlation between separate data sets. We used a simple logistic regression to determine the probability of detecting VNA with our $\mathrm{cVNT}$ as a function of the anti-SARS-CoV-2 IgG or sVNT results.

\section{Results}

This study included 59 individuals. Nine and eight of them received a homologous immunisation with AZD1222 or BNT162b2, respectively, while 42 received a heterologous vaccination. The composition of the study groups including median age, age range, gender, and median time of blood collection in days is shown in Table 1. 
After the first immunisation, all individuals who received an mRNA vaccine developed anti-(trimeric)-S and anti-RBD-IgG. The vaccinees who received the AZD1222 had a response rate of $55.3 \%$ (anti-S IgG), 76.3\% (anti-trimeric S IgG), and 94.7\% (anti-RBD IgG), respectively. After administration of the second dose, the IgG response rate reached $100 \%$ in all groups. The median of anti-SARS-CoV-2 IgG concentrations varied between $20.9 \mathrm{BAU} / \mathrm{ml}$ (anti-S IgG; first vaccination in the AZD1222 heterologous group) and $6240 \mathrm{BAU} / \mathrm{ml}$ (antitrimeric S IgG; second vaccination in the BNT162b2 homologous group). After the second vaccine dose, an increase in median anti-SARS-CoV-2 IgG concentrations was observed in all three study groups and in all assays. Compared to the median anti-SARS-CoV-2 IgG concentrations after the vector vaccine AZD1222 was administered twice, the corresponding concentrations were 6 to 12 times higher after a heterologous vaccination and even 11 to 20 times higher when compared to the homologous BNT162b2 vaccination scheme (Fig. 1; Additional file 1: Table S1).

Next, we examined the presence of IgG directed against the separate NPs of seasonal HCoVs and SARSCoV-2 as well as against the S1 and RBD of SARS-CoV2 in an immunoblot. Between 19.6 and $38.0 \%$ of the vaccinees had IgG antibodies that were directed against the NPs of seasonal HCoVs, while none showed IgG reactivity against the NP of SARS-CoV-2. The second vaccine dose resulted in a $100 \%$ prevalence of anti-S1 and antiRBD IgG antibodies (Additional file 1: Fig. S1). Furthermore, development of anti-SARS-CoV-2 IgG avidity was recorded. After the first vaccination, the majority of the measured IgG avidity indices were in the low to intermediate range. In contrast, high IgG avidities were consistently observed after administration of the second vaccine dose (Fig. 2; Additional file 1: Table S1).

The virus-neutralising properties of the sera were examined with two different assays. A so-called surrogate

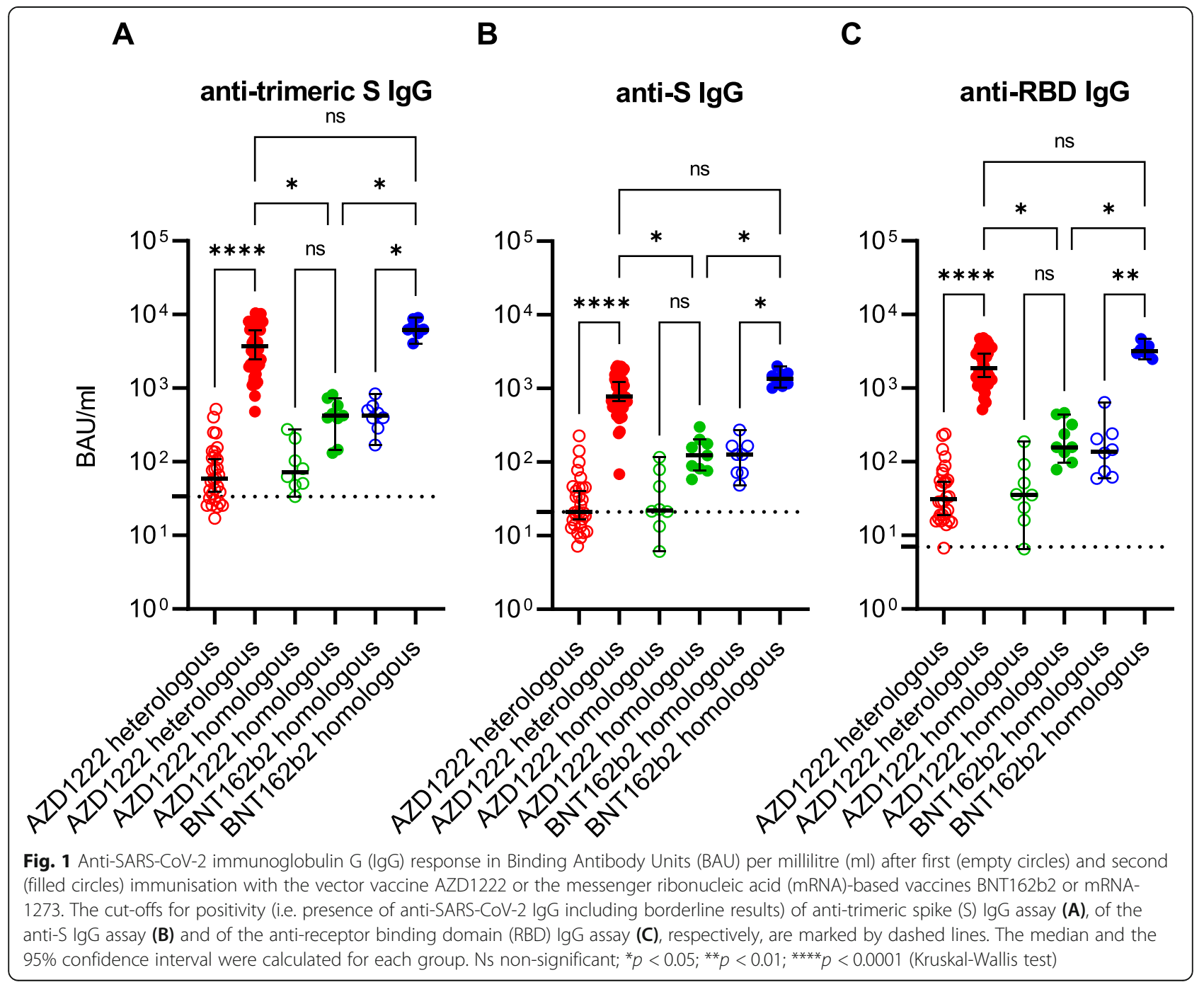




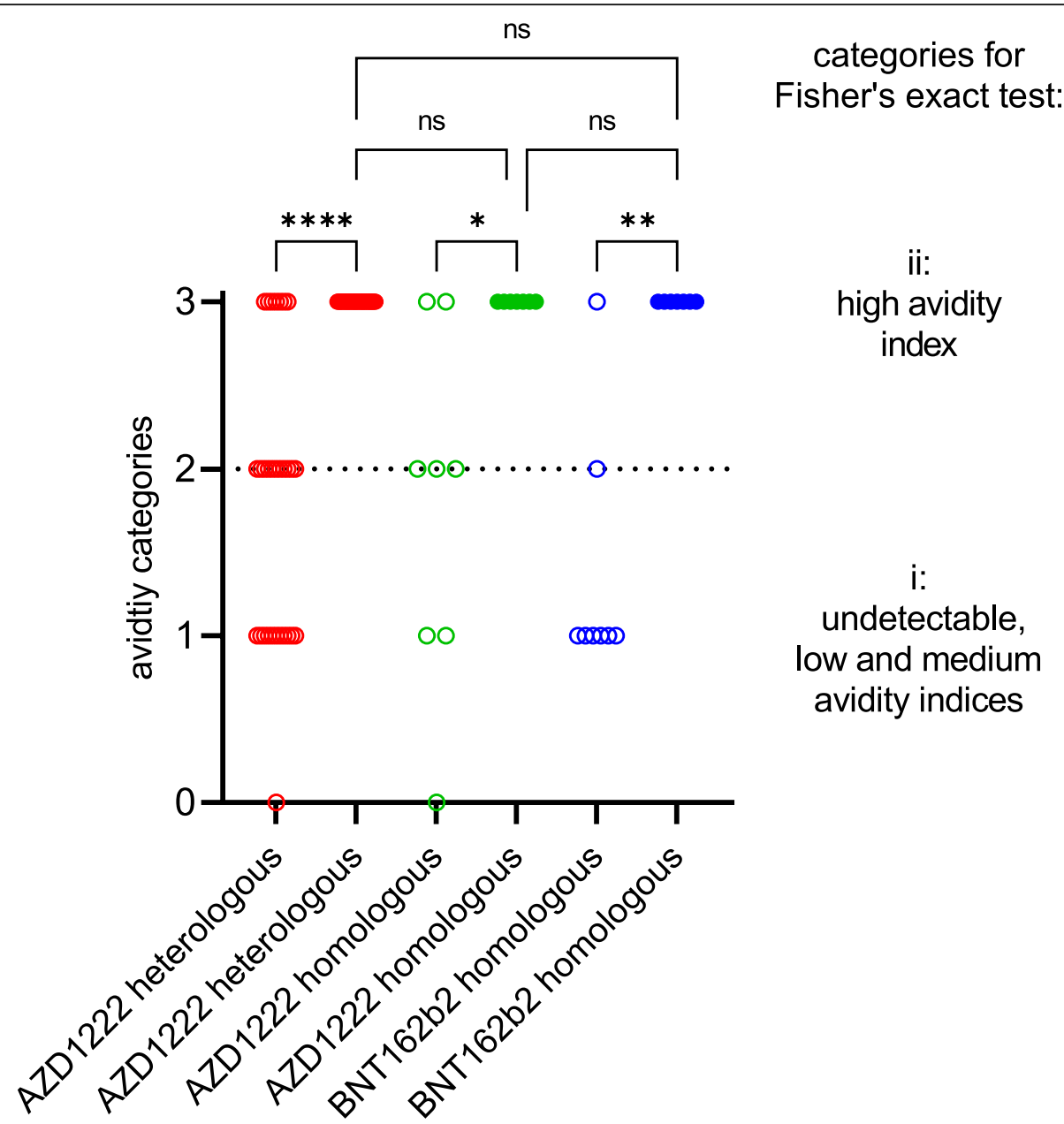

Fig. 2 Development of anti-SARS-CoV-2 immunoglobulin G avidities after first (empty circles) and second (filled circles) immunisation with the vector vaccine AZD1222 or the messenger ribonucleic acid (mRNA)-based vaccines BNT162b2 or mRNA-1273. The measured IgG avidities were assigned to the four categories of undetectable (0), low (1), intermediate (2) and high (3) index. The significance of the distribution differences was calculated between the two groups of undetectable, low and intermediate (i) on the one hand and high (ii) avidity indices on the other. Ns not significant; ${ }^{*} p<0.02 ;{ }^{* *} p<0.003 ;{ }^{* * *} p<0.00003$ (Bonferroni-adjusted Fisher's exact test)

neutralisation test was used to investigate the extent to which the anti-RBD antibodies that may be present in the serum are able to prevent the binding of this $\mathrm{S}$ protein subunit to the human receptor ACE-2. In addition, a laboratory-developed virus-neutralisation test was used, which is based on a VOC B.1.1.7 strain as the antigen. While the sVNT classified the majority of the sera as virus-neutralising after the first vaccination, most of these samples in the cVNT were below the cut-off from which virus neutralisation can be assumed. With both methods, however, an increase in the level of VNA could be detected after a second vaccination. There were also marked differences between the three vaccination groups, both in the degree of inhibition (sVNT) and in the level of VNA titres (cVNT). Vaccinees who had received the vector vaccine only had 11-fold lower median VNA titres (1:57) compared to individuals immunised heterologously with AZD1222 and an mRNA vaccine (1: $640)$ or homologously with BNT162b2 (1:640). In contrast, the median percentage inhibition of sVNT reached a similar level in all three groups (Fig. 3; Additional file 1: Table S1). The quantitative results of the anti-SARSCoV-2 IgG assays showed an almost perfect correlation to the VNA titres using VOC B.1.1.7 as antigen in the cVNT (Spearman correlation coefficient of 0.86 to 0.88 ; Fig. $4 \mathrm{~A}-\mathrm{C}$ ). In addition, from a certain anti-SARS-CoV2 IgG concentration, it could be assumed with a probability of $95 \%$ that VNA are present. The anti-SARS$\mathrm{CoV}-2$ IgG BAU/ml concentrations required for this differed slightly between the three assays $(323 \mathrm{BAU} / \mathrm{ml}$ in the anti-S IgG test; $448 \mathrm{BAU} / \mathrm{ml}$ in the anti-RBD IgG test; $886 \mathrm{BAU} / \mathrm{ml}$ in the anti-trimeric S IgG test). However, there is a large overlap of samples that are virusneutralising or not at a similar IgG concentration (Fig. 


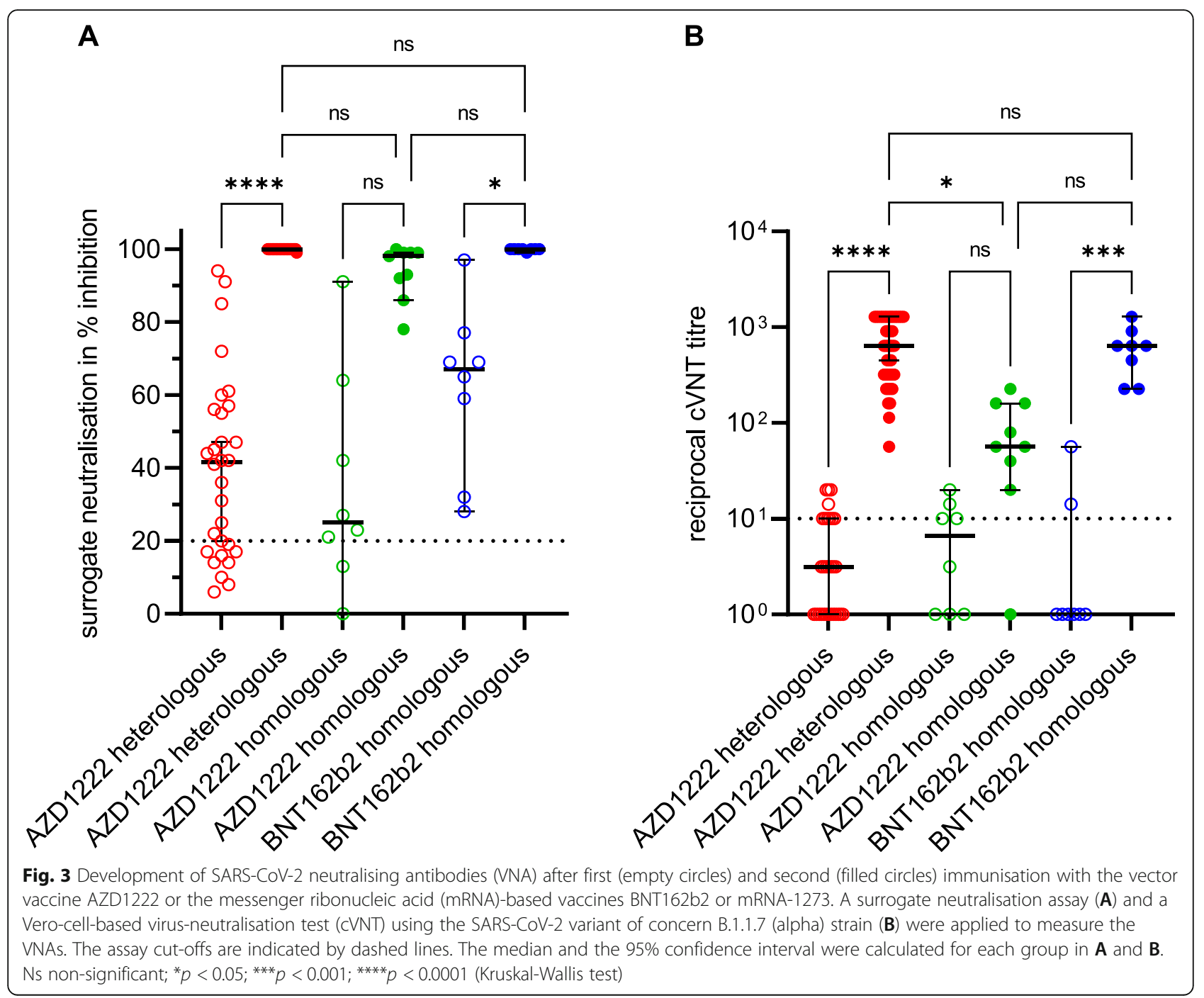

4D-F). The correlation between the measured values of cVNT (VOC B.1.1.7 as antigen) and sVNT was comparable (Spearman correlation coefficient of 0.88 ) to those calculated for the three IgGs. It is noticeable that especially non-neutralising sera (VNA titre $\leq 1: 10$ ) were overestimated in the sVNT. This is evidenced by the fact that the cut-off set by the manufacturer was only associated with a $4 \%$ probability of detecting VNA in our cVNT (Fig. 5).

A subset of age- and gender-matched sera (Table 1) obtained after the second immunisation were also tested in the cVNT for the presence of VNA against VOC B.1.617.2 (delta). In comparison to B.1.1.7, all three vaccination groups exhibited significantly lower median VNA titres (AZD1222/BNT162b2: 1:1280 vs. 1:80, 16fold lower; AZD1222/AZD1222: 1:57 vs. 1:20, 2.9-fold lower; BNT162b2/BNT162b2: 1:640 vs. 1:160, 4-fold lower) when B.1.617.2 is used as the antigen. If the vaccination schedule is used as a comparator, those vaccinated homologously with the vector vaccine had significantly lower median VNA titres (1:20) than those homologously vaccinated with BNT162b2 (1:160). This difference was also found for the heterologous vaccination regime $(1: 80)$. In contrast, the median VNA titres between those vaccinated homologously with BNT162b and those vaccinated heterologously (AZD1222/ BNT162b2) exhibited no significant difference (Fig. 6; Additional file 1: Table S1).

\section{Discussion}

Due to the rare but serious side effects after administration of the vector vaccine (AZD1222), in spring 2021, the STIKO recommended that people under the age of 60 should complete vaccinations that had already been started with a vector vaccine with an mRNA vaccine $[9$, 10]. This recommendation was extended on July 1,2021 , to all who had already received a primary vaccination with AZD1222 [12]. In the first quarter of this year, 


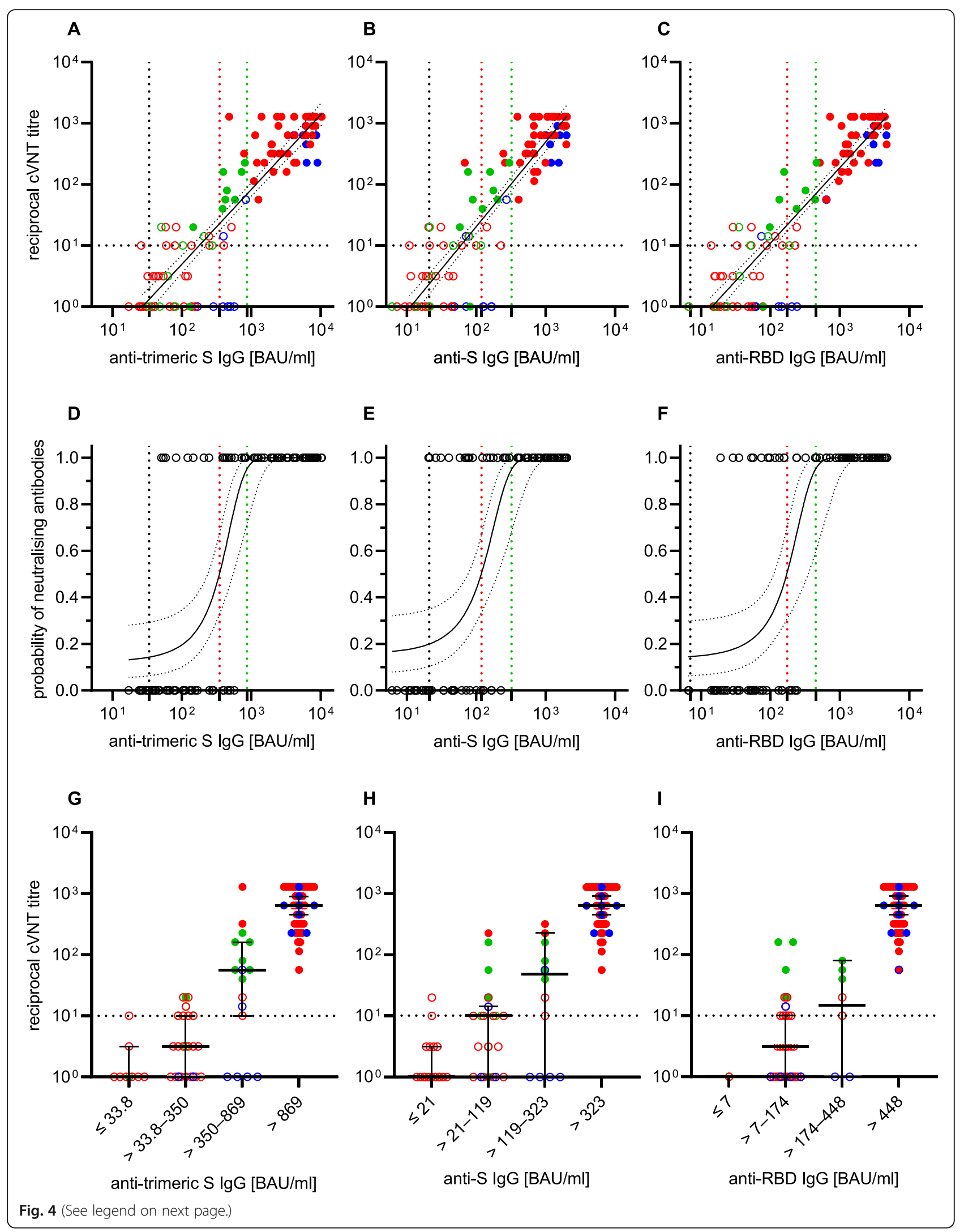


(See figure on previous page.)

Fig. 4 Anti-SARS-CoV-2 immunoglobulin G (lgG) response in Binding Antibody Units (BAU) per millilitre (ml) after first (open circles) and second (filled circles) immunisation with the vector vaccine AZD1222 (green), the messenger ribonucleic acid (mRNA)-based vaccine BNT162b2 (blue) and after a heterologous vaccination scheme, starting with AZD1222, followed by an mRNA-based vaccine boost (BNT162b2 or mRNA-1273; red) with regard to the detection of virus-neutralising antibodies (VNA). The latter were measured in a Vero-cell-based neutralisation test (cVNT) using the SARS-CoV-2 variant of concern B.1.1.7 (alpha). Cut-off values for positivity of the anti-trimeric spike (S) IgG assay (A), anti-S IgG assay (B) and anti-receptor binding domain (RBD) IgG assay $(\mathbf{C})$, respectively, and the cVNT cut-off value for the presence of VNA are indicated by black dashed lines. The Spearman correlation coefficients of log(reciprocal titre) were calculated with $0.86,0.86$ and 0.88 , respectively. The probability of detecting VNA at a given BAU/ml in the anti-SARS-CoV-2 lgG assays was calculated by logistic regression (D-F): VNA were present in 95\% of samples when lgG concentrations of $886 \mathrm{BAU} / \mathrm{ml}$ (anti-trimeric S lgG), $323 \mathrm{BAU} / \mathrm{ml}$ (anti-S lgG) and $448 \mathrm{BAU} / \mathrm{ml}$ (anti-RBD lgG), respectively, were measured (green dashed lines; $95 \%$ confidence intervals (CI) 59.4 to 99.6\%). Vertical black dashed lines represent the threshold values set by the manufacturers of the antibody assay; red dashed lines represent the BAU/ml concentrations (anti-trimeric S lgG: 350 BAU/ml; anti-S lgG: 119 BAU/ $\mathrm{ml}$; anti-RBD IgG: $174 \mathrm{BAU} / \mathrm{ml}$ ) with a 50\% probability of VNA detection. The distribution of the CVNT titres, the medians, and the $95 \% \mathrm{Cls}$ between the three plotted thresholds (dashed black, red and green lines in $\mathbf{A}-\mathbf{F}$ ) are shown $(\mathbf{G}-\mathbf{I}$ )

however, only a few animal experimental data sets were available on the immunological outcome of the proposed heterologous vaccination scheme $[13,14]$. Several studies have now appeared on the immunogenicity of such immunisation schemes in humans [16-22, 29].

In this report, we compared the development of the humoral immune response after homologous and heterologous vaccination with different methods. To the best of our knowledge, we are one of the first research groups to investigate the anti-delta VOC neutralising effect of sera after completing the heterologous immunisation regime.

After the first vaccination, the majority of individuals developed anti-trimeric-S, anti-S, and anti-RBD IgG antibodies, respectively. However, their concentrations varied between the three study groups. The results are in line with our previous study [23]. It is evident that the anti-SARS-CoV-2 IgG concentrations are not comparable between the three assays either. This is probably due to the different antigen preparations. In order to make valid statements about the kinetics of the antibody concentration in individuals or to compare the antibody response between different groups, identical assays should preferably be used; alternatively, the inclusion of reference standards could be useful.

The second immunisation resulted in higher concentrations in all three groups. It was noticeable that significantly higher anti-SARS-CoV-2 IgG concentrations were detected after a second vaccination with an mRNA vaccine than after the vector vaccine AZD1222 was

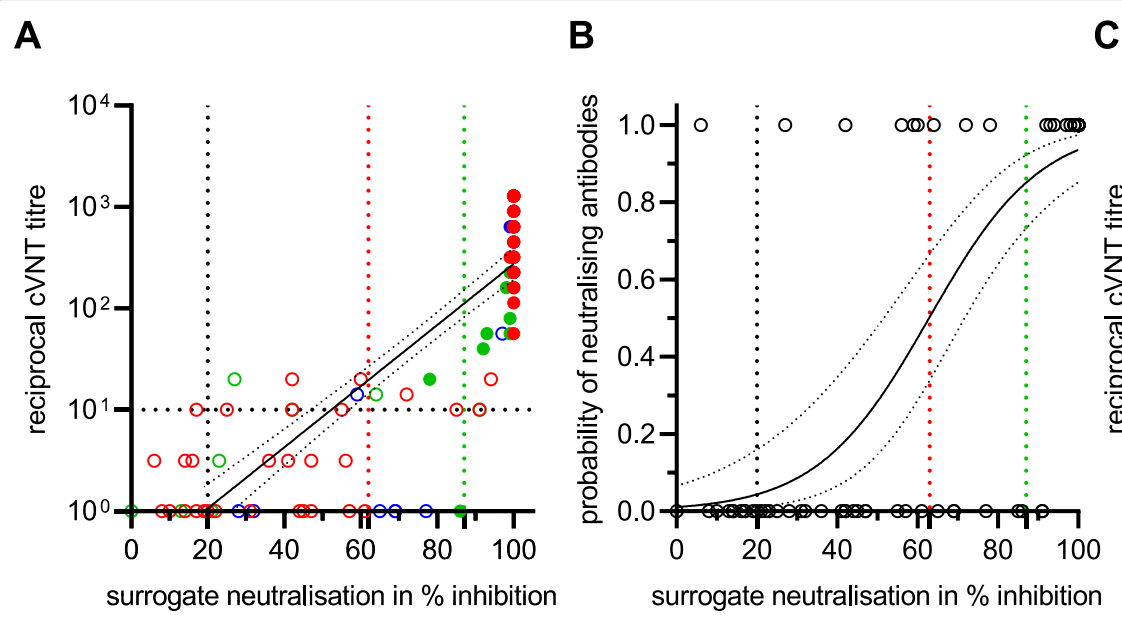

C

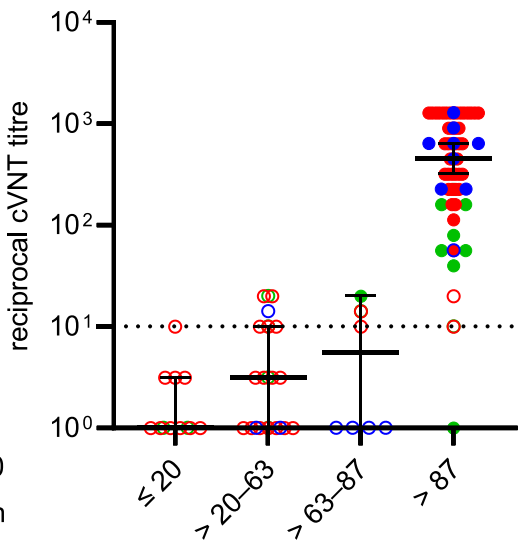

surrogate neutralisation in \% inhibition

Fig. 5 Correlation of the surrogate neutralisation test (sVNT) results with results obtained by the laboratory-developed Vero-cell-based virusneutralisation test (cVNT) using a B.1.1.7 strain as antigen (A). The Spearman correlation coefficient of log(reciprocal titre) was calculated with 0.88; empty circles: first vaccination; filled circles: second vaccination; red: heterologous vaccination with AZD1222/mRNA; green: homologous vaccination with AZD1222; blue: homologous vaccination with BNT162b2. Probability of detecting virus-neutralising antibodies (VNA) with the cVNT at a given percentage inhibition of sVNT calculated by logistic regression (B); e.g. at 20\% inhibition (black dashed line), 63\% inhibition (red dashed line), and at $87 \%$ inhibition of sVNT (green dashed line), the probabilities of detecting VNA with cVNT are $4 \%$ (95\% confidence interval (CI) 1-16\%), $50 \%$ (95\% Cl 34-66 \%) and 85\% (95\% Cl 73-92\%), respectively. The distribution of the CVNT titres, their medians, and their 95\% Cls between the three plotted thresholds (dashed black, red and green lines in $\mathbf{A}, \mathbf{B}$ ) are shown (C) 


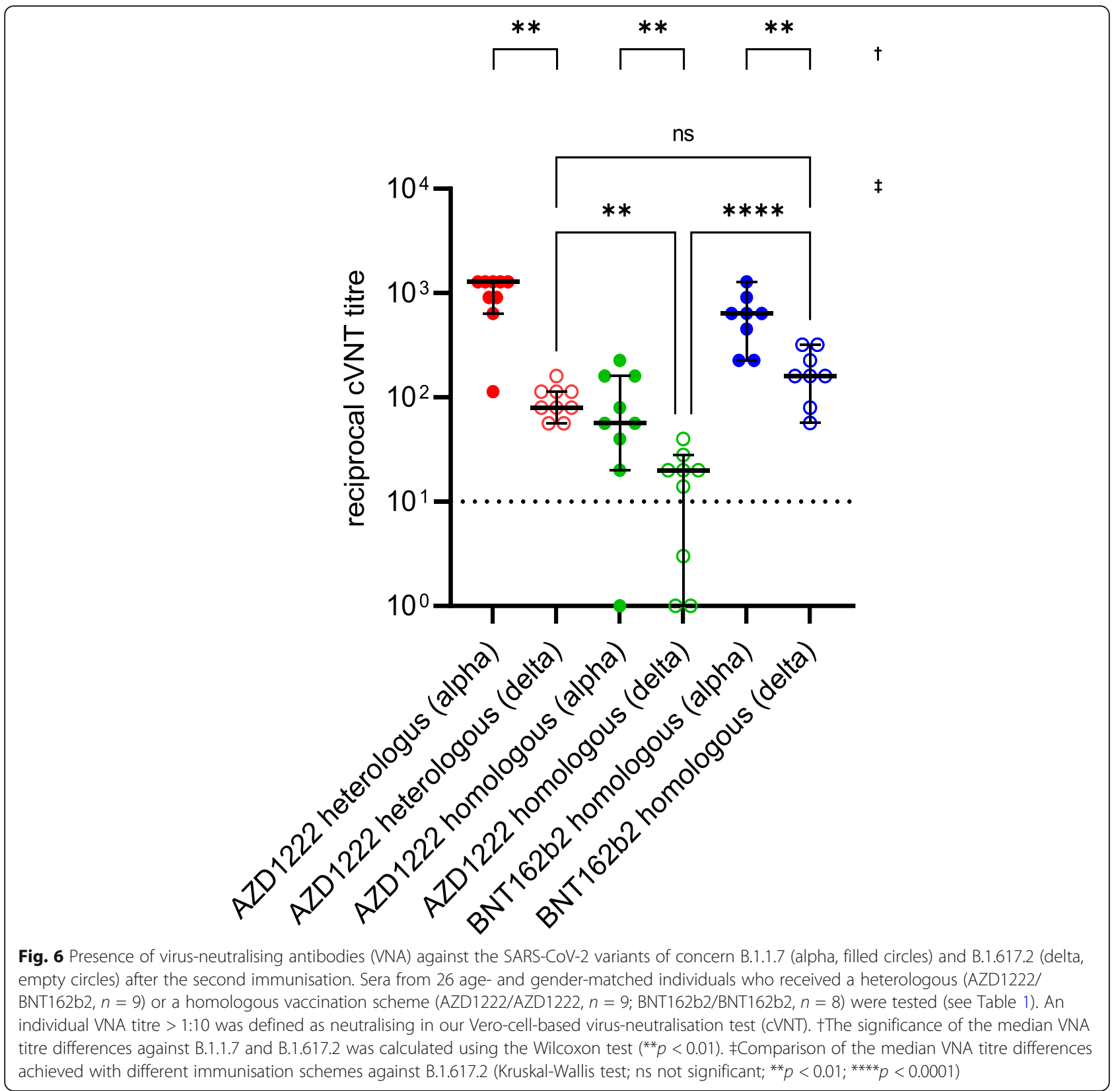

administered again. The increase in anti-S and anti-RBD IgG concentrations after a second vaccination with an mRNA vaccine confirms our previous study [23]. Due to the recommended vaccination interval of 10 to 12 weeks, we did not yet have any data on the development of the SARS-CoV-2-specific IgG antibodies after the second administration of a vector vaccine [23]. The lack of antiSARS-CoV-2 NP-specific IgG antibodies in all vaccinees can be interpreted as an indication that they had no COVID-19 infection and were therefore to be regarded as immunologically naive before immunisation [23]. It is known that vaccinations lead to particularly high antiSARS-CoV-2 IgG concentrations in convalescents [30].
For these individuals, the recommendation is that they should receive a vaccine dose about 6 months after they have been infected [10]. Less than half of the vaccinees had IgG antibodies to the NPs of seasonal coronaviruses, which is lower than the prevalence reported by other authors for adults [31].

After the first vaccination, nearly all individuals exhibited only low to intermediate avid anti-SARS-CoV-2 IgG, while after the second vaccine dose IgG of high avidity appeared in all cases. These results confirm and expand the existing knowledge on the development of highly avid anti-SARS-CoV-2 IgG after a second vaccination with an mRNA vaccine [32]. In line with this, 
VNA titres $>1: 10$ against the previously prevalent SARS-CoV-2 VOC B.1.1.7 (alpha) were observed after second immunisation which confirms our recent study [23]. Marked differences in median VNA titres were observed between individuals re-vaccinated with an mRNA vaccine and those re-vaccinated with the vector vaccine. The anti-SARS-CoV-2 IgG concentrations obtained with three preparations of the viral S-protein correlated well with the presence and level of VNA titres using B.1.1.7 as the antigen in our cVNT. This observation is in line with a recently published study from Finland [33] and suggests that these standardised and easy-to-perform commercial tests are useful for measuring vaccine-induced responses. High anti-SARS-CoV2 IgG concentrations were associated with the presence of high VNA titres against VOC B.1.1.7 (alpha). However, anti-SARS-CoV-2 IgG concentrations that indicate the presence of protective VNA cannot be defined across the board. These depend on the cVNT and the viral antigen used in it. Basically, the sVNT also showed the titre increase and evaluated all sera as virusneutralising after the second vaccination with values close to $100 \%$ inhibition of RBD binding to ACE-2. However, it is again noticeable that sera that are not or only weakly SARS-CoV-2 neutralising in the in-house cVNT are categorised as neutralising in the sVNT, which supports our previous proposal to increase the cut-off of this surrogate assay [23]. Compared to the admittedly very conservative in-house cVNT, a cut-off of over $80 \%$ binding inhibition would be desirable. Due to the lack of standardisation of the widespread cVNTs for the detection of VNA against SARS-CoV-2, this recommendation only applies to our laboratory and cannot be generalised.

A particularly interesting point is the significantly reduced capacity for neutralising the SARS-CoV-2 delta variant (VOC B.1.617.2) in vitro using a cVNT. We observed this in the subgroup of 26 age- and gendermatched individuals regardless of the immunisation regime. In addition, three of nine vaccinees who had received two doses of AZD1222 presented low or undetectable VNA against this VOC, which is considered to be $60 \%$ more transmissible than alpha [34]. The SARS-CoV-2 delta VOC is known to have accumulated a number of mutations in the S-protein. These enable continued good binding to the cellular ACE-2 receptor, but at the same time lead to the viral S-protein being less efficiently recognised by antibodies [34]. The significantly lower VNA titres compared to the alpha VOC, which we and others [34, 35] observed, corroborate the suspicion of an immunescape of the delta VOC. The results of our cVNT for B.1.1.7 suggest that a single vaccination would not be sufficient to induce measurable VNA against B.1.617.2.
The data presented by us on the antibody response after heterologous SARS-CoV-2 vaccination are consistent with the few available clinical studies [16-22, 29]. In June 2021, a randomised study from Spain has already demonstrated that the heterologous vaccination scheme is suitable for generating a robust immune response. Unfortunately, this very extensive work did not include a control group of individuals who received two immunisations with the vector vaccine [21]. A recent preprint reports significant higher anti-S antibody concentrations in a group of 26 individuals who first received an AZD1222 vaccination followed by re-vaccination with BNT162b2 compared to 14 individuals that were vaccinated twice with BNT162b2. However, these results were obtained with a total antibody assay which does not discriminate between IgG and acute phase immunoglobulin $\mathrm{M}$. The VNA titres against chimeric vesicular stomatitis viruses carrying the S-proteins of SARS-CoV2 VOCs B.1.1.7 (alpha) or B.1.351 (beta) as antigens, respectively, were markedly higher in the AZD1222/ BNT162b2 group compared to the BNT162b2/ BNT162b2 recipients, while data after homologous vaccination with the vector vaccine were not presented [16]. A further study, however, which also includes results from individuals who were vaccinated twice with AZD1222, came to the same conclusion [22]. In another investigation [18], differences in the anti-S IgG concentrations and VNA titres were not observed after heterologous vaccination (AZD1222/mRNA) or after homologous vaccination with an mRNA vaccine while both parameters were significantly lower after homologous vaccination with the vector vaccine. These results are in agreement with our data even if only one IgG assay and one sVNT were used by this research group [18]. The data of a single-blind randomised British study, in which the four possible vaccine combinations of AZD1222 and BNT162b2 were compared with one another, are very interesting and promising. These researchers report about 9-fold higher geometric mean anti-S IgG concentrations in sera from heterologous AZD1222/BNT162b2 vaccinees compared to individuals immunised twice with AZD1222 [19]. This largely corresponds to our results and to data of a current preprint, which describes the immune response after administration of combinations of vector (Ad26.COV2 - S, Janssen) and mRNA (BNT162b2, mRNA-1273) vaccines [29]. In a study from Sweden, markedly higher anti-S and antiRBD IgG concentrations were observed after heterologous vaccination (AZD1222/mRNA-1273) compared to the homologous AZD1222 scheme. Likewise, significantly higher VNA titres were measured both against a wild-type SARS-CoV-2 and against a VOC strain B.1.351 (beta). Two doses of AZD1222, however, did not induce potent VNA titres against the beta VOC [20] as also 
observed by others [22]. One investigation reports the development of high IgG avidity after completion of a homologous (AZD1222/AZD1222; BNT162b2/ BNT162b2) or heterologous SARS-CoV-2 (AZD1222/ BNT162b2) immunisation scheme. While there were no qualitative differences in the development of IgG avidity between both groups, the AZD1222/BNT162b2 vaccinees developed a significantly higher relative avidity index [17]. In our IgG avidity assay, we cannot measure such gradual differences. For the AZD1222/AZD1222 group, lower anti-SARS-CoV-2 IgG concentrations as well as lower B.1.1.7/B.1.351-pseudovirus-neutralising titres were reported [17].

It is not yet sufficiently clear why homologous vaccination with the AZD1222 vector vaccine leads to lower anti-SARS-CoV-2 IgG concentrations and VNA titres. A possible explanation could be the immune response to the adenovirus vector backbone (so-called antivector immunity [3]).

The work presented by us contributes to a better understanding of the humoral immunogenicity of the heterologous SARS-CoV-2 vaccination regimen. With various assays, we monitored the development of anti-Sspecific IgG antibodies and make statements about their binding strength as an expression of maturity. In addition, with a commercial and an in-house test, we showed that VNA can be detected after the second vaccination and that VNA titres vary in dependence of the viral antigen.

Important limitations of our report are (i) the heterogeneity of the study groups; (ii) the marked difference in time between the first immunisation and sampling which may impair data comparability; (iii) the small group size of individuals who received a homologous vaccination scheme; (iv) the subjects, who are predominantly in younger to middle adulthood; (v) the lack of information on the durability of the detected antibodies; and (vi) the missing consideration of cellular and innate immunity after immunisation. Therefore, no statements can be made about the need for further booster vaccinations. In addition, our data on anti-SARS-CoV-2 IgG concentrations do not allow any valid predictions about the degree of protection against natural SARS-CoV-2 infection.

\section{Conclusions}

The administration of a vector vaccine followed by an mRNA vaccine boost resulted in a strong humoral immune response, comparable to that after two immunisations with an mRNA vaccine. Regardless of the vaccination scheme, all individuals developed highly avid anti-SARS-CoV-2 IgGs as well as VNA against a B.1.1.7 strain (alpha VOC) after the second immunisation. However, the generally lower neutralising titres against the B.1.617.2 strain, which were observed in the subgroup of
26 vaccinees demonstrate a partial immune escape of the delta VOC. While these results require further confirmation, they suggest that adapting the vaccine to current virus variants may be useful.

\section{Abbreviations \\ ACE-2: Angiotensin-converting enzyme 2; AZD1222: Vector vaccine from AstraZeneca (ChAdOx1, Vaxzevria); BAU: Binding Antibody Units; BNT162b2: Messenger ribonucleic acid vaccine from Pfizer/BioNTech; cVNT: Vero-cell-based neutralisation test; IgG: Immunoglobulin G; m- 1273: Messenger ribonucleic acid vaccine from Moderna; mRNA: Messenger ribonucleic acid; NP: Nucleoprotein; RBD: Receptor binding domain; SARS- CoV-2: Severe acute respiratory syndrome coronavirus 2; S-protein: Spike protein; STIKO: Standing Vaccination Commission of the Robert Koch Institute; SVNT: Surrogate neutralisation test; VNA: Virus-neutralising antibodies; VOC: Variant of concern; WHO: World Health Organization}

\section{Supplementary Information}

The online version contains supplementary material available at https://doi. org/10.1186/s12916-021-02231-x

Additional file 1. Table S1: Vaccine-induced humoral immunity. Fig. S1: Nucleoprotein (NP) specific immune response.

\section{Acknowledgements}

The authors would like to thank all volunteers for participating in the study.

\section{Authors' contributions}

AK, FN and RR had full access to all the data in the study and take responsibility for the integrity of the data and the accuracy of the data analysis. Concept and design: AK. Acquisition, analysis or interpretation of the data: all authors. Drafting of the manuscript: AK, HF and RR. Critical revision of the manuscript for important intellectual content: all authors. All authors have read and agreed to the final version of the manuscript

\section{Funding}

This research received no external funding. Open Access funding enabled and organized by Projekt DEAL.

Availability of data and materials

The manuscript and Additional file 1 contain all relevant data from the study.

\section{Declarations}

\section{Ethics approval and consent to participate}

The study was conducted according to the guidelines of the Declaration of Helsinki and approved by the Ethics Committee of the medical faculty of the Christian-Albrechts-Universität zu Kiel, Kiel, Germany (D467/20, 16.04.2020; amendment 02.02.2021). Informed consent was obtained from all subjects involved in the study.

\section{Consent for publication}

Not applicable.

\section{Competing interests}

The companies Abbott $\mathrm{GmbH}$, Diasorin $\mathrm{GmbH}$, Mikrogen $\mathrm{GmbH}$ and Tecomedical $\mathrm{GmbH}$ supported this study by providing free or discounted kits. None of the four companies had any influence on the testing and the interpretation of the results. The authors declare that they have no competing interests.

Received: 16 September 2021 Accepted: 30 December 2021 Published online: 21 January 2022

References

1. Hu B, Guo H, Zhou P, Shi ZL. Characteristics of SARS-CoV-2 and COVID-19. Nat Rev Microbiol. 2021;19(3):141-54. https://doi.org/10.1038/s41579-02000459-7. 
2. Klasse PJ, Nixon DF, Moore JP. Immunogenicity of clinically relevant SARS CoV-2 vaccines in nonhuman primates and humans. Sci Adv. 2021;7(12): eabe8065. https://doi.org/10.1126/sciadv.abe8065.

3. Sadarangani M, Marchant A, Kollmann TR. Immunological mechanisms of vaccine-induced protection against COVID-19 in humans. Nat Rev Immunol. 2021;21(8):475-84. https://doi.org/10.1038/s41577-021-00578-z.

4. Krammer F. SARS-CoV-2 vaccines in development. Nature. 2020;586(7830): 516-27. https://doi.org/10.1038/s41586-020-2798-3.

5. Cines DB, Bussel JB. SARS-CoV-2 vaccine-induced immune thrombotic thrombocytopenia. N Engl J Med. 2021;384(23):2254-6. https://doi.org/10.1 056/NEJMe2106315.

6. Kyriakidis NC, Lopez-Cortes A, Gonzalez EV, Grimaldos AB, Prado EO. SARS CoV-2 vaccines strategies: a comprehensive review of phase 3 candidates. NPJ Vaccines. 2021;6(1):28. https://doi.org/10.1038/s41541-021-00292-w.

7. Creech CB, Walker SC, Samuels RJ. SARS-CoV-2 vaccines. JAMA. 2021;325(13): 1318-20. https://doi.org/10.1001/jama.2021.3199.

8. EU. Safe COVID-19 vaccines for Europeans. 2021. https://ec.europa.eu/info/ live-work-travel-eu/coronavirus-response/safe-covid-19-vaccines-europeans en. Accessed 12 May 2021.

9. STIKO. Stellungnahme der Ständigen Impfkommission zum Zeitpunkt der Gabe eines mRNA-Impfstoffs nach Erstimpfung mit AstraZeneca Vaccine (Vaxzevria) bei <60-Jährigen. 2021. https://www.rki.de/DE/Content/ Kommissionen/STIKO/Empfehlungen/Stellungnahme-Impfabstand.html. Accessed 12 May 2021

10. RKI. Mitteilung der Ständigen Impfkommission beim Robert Koch-Institut Beschluss der STIKO zur 7. Aktualisierung der COVID-19-Impfempfehlung und die dazugehörige wissenschaftliche Begründung. Epidemiol Bull. 2021; 25:3-13.

11. Rambaut A, Holmes EC, O'Toole A, Hill V, McCrone JT, Ruis C, et al. A dynamic nomenclature proposal for SARS-CoV-2 lineages to assist genomic epidemiology. Nat Microbiol. 2020;5(11):1403-7. https://doi.org/10.1038/s41 564-020-0770-5.

12. STIKO. Mitteilung der STIKO zur COVID-19-Impfung: Impfabstand und heterologes Impfschema nach Erstimpfung mit Vaxzevria (1.7.2021). 2021. https://www.rki.de/DE/Content/Kommissionen/STIKO/Empfehlungen/PM_2 021-07-01.html. Accessed 2 July 2021.

13. Spencer AJ, McKay PF, Belij-Rammerstorfer S, Ulaszewska M, Bissett CD, Hu $K$, et al. Heterologous vaccination regimens with self-amplifying RNA and adenoviral COVID vaccines induce robust immune responses in mice. Nat Commun. 2021;12(1):2893. https://doi.org/10.1038/s41467-021-23173-1.

14. He Q, Mao Q, An C, Zhang J, Gao F, Bian L, et al. Heterologous prime-boost: breaking the protective immune response bottleneck of COVID-19 vaccine candidates. Emerg Microbes Infect. 2021;10(1):629-37. https://doi.org/10.1 080/22221751.2021.1902245.

15. Shaw RH, Stuart A, Greenland M, Liu X, Nguyen Van-Tam JS, Snape MD, et al. Heterologous prime-boost COVID-19 vaccination: initial reactogenicity data. Lancet. 2021;397(10289):2043-6. https://doi.org/10.1016/S0140-6736(21 )01115-6.

16. Groß R, Zanoni M, Seidel A, Conzelmann C, Gilg A, Krnavek D, et al. Heterologous ChAdOx1 nCoV-19 and BNT162b2 prime-boost vaccination elicits potent neutralizing antibody responses and T cell reactivity. medRxiv. 2021;75:103761. https://doi.org/10.1101/2021.05.30.21257971.

17. Hillus D, Schwarz T, Tober-Lau P, Vanshylla K, Hastor H, Thibeault C, et al. Safety, reactogenicity, and immunogenicity of homologous and heterologous prime-boost immunisation with ChAdOx1 nCoV-19 and BNT162b2: a prospective cohort study. Lancet Respir Med. 2021;9(11):125565. https://doi.org/10.1016/S2213-2600(21)00357-X.

18. Schmidt T, Klemis V, Schub D, Mihm J, Hielscher F, Marx S, et al Immunogenicity and reactogenicity of heterologous ChAdOx 1 nCoV-19/ mRNA vaccination. Nat Med. 2021;27(9):1530-5. https://doi.org/10.1038/s41 591-021-01464-w.

19. Liu X, Shaw RH, Stuart ASV, Greenland M, Aley PK, Andrews NJ, et al. Safety and immunogenicity of heterologous versus homologous prime-boost schedules with an adenoviral vectored and mRNA COVID-19 vaccine (ComCOV): a single-blind, randomised, non-inferiority trial. Lancet. 2021; 398(10303):856-69. https://doi.org/10.1016/S0140-6736(21)01694-9.

20. Normark J, Vikstrom L, Gwon YD, Persson IL, Edin A, Bjorsell T, et al. Heterologous ChAdOx1 nCoV-19 and mRNA-1273 vaccination. N Engl J Med. 2021;385(11):1049-51. https://doi.org/10.1056/NEJMc2110716.

21. Borobia AM, Carcas AJ, Perez-Olmeda M, Castano L, Bertran MJ, Garcia-Perez J, et al. Immunogenicity and reactogenicity of BNT162b2 booster in
ChAdOx1-S-primed participants (CombiVacS): a multicentre, open-label, randomised, controlled, phase 2 trial. Lancet. 2021;398(10295):121-30. https://doi.org/10.1016/S0140-6736(21)01420-3.

22. Barros-Martins J, Hammerschmidt SI, Cossmann A, Odak I, Stankov MV, Morillas Ramos G, et al. Immune responses against SARS-CoV-2 variants after heterologous and homologous ChAdOx1 nCoV-19/BNT162b2 vaccination. Nat Med. 2021;27(9):1525-9. https://doi.org/10.1038/s41591-021-01449-9.

23. Neumann F, Rose R, Rompke J, Grobe O, Lorentz T, Fickenscher H, et al. Development of SARS-CoV-2 specific lgG and virus-neutralizing antibodies after infection with variants of concern or vaccination. Vaccines (Basel). 2021;9(7):700. https://doi.org/10.3390/vaccines9070700.

24. Bonelli F, Blocki FA, Bunnell T, Chu E, De La OA, Grenache DG, et al. Evaluation of the automated LIAISON((R)) SARS-CoV-2 TrimericS IgG assay for the detection of circulating antibodies. Clin Chem Lab Med. 2021;59(8): 1463-7. https://doi.org/10.1515/cclm-2021-0023.

25. Kristiansen PA, Page M, Bernasconi V, Mattiuzzo G, Dull P, Makar K, et al. WHO International Standard for anti-SARS-CoV-2 immunoglobulin. Lancet. 2021;397(10282):1347-8. https://doi.org/10.1016/S0140-6736(21)00527-4.

26. Stromer A, Rose R, Grobe O, Neumann F, Fickenscher H, Lorentz T, et al. Kinetics of nucleo- and spike protein-specific immunoglobulin $\mathrm{G}$ and of virus-neutralizing antibodies after SARS-CoV-2 infection. Microorganisms. 2020:8(10):1572. https://doi.org/10.3390/microorganisms8101572.

27. Stromer A, Rose R, Schafer M, Schon F, Vollersen A, Lorentz T, et al. Performance of a point-of-care test for the rapid detection of SARS-CoV-2 antigen. Microorganisms. 2020;9(1):58. https://doi.org/10.3390/microorga nisms9010058.

28. Earle KA, Ambrosino DM, Fiore-Gartland A, Goldblatt D, Gilbert PB, Siber GR, et al. Evidence for antibody as a protective correlate for COVID-19 vaccines. Vaccine. 2021;39(32):4423-8. https://doi.org/10.1016/j.vaccine.2021.05.063.

29. Atmar RL, Lyke KE, Deming ME, Jackson LA, Branche AR, El Sahly HM, et al. Heterologous SARS-CoV-2 booster vaccinations - preliminary report. medRxiv. 2021. https://doi.org/10.1101/2021.10.10.21264827.

30. Frieman $M$, Harris $A D$, Herati RS, Krammer F, Mantovani $A$, Rescigno $M$, et al. SARS-CoV-2 vaccines for all but a single dose for COVID-19 survivors. EBioMedicine. 2021;68:103401. https://doi.org/10.1016/j.ebiom.2021.103401.

31. Severance EG, Bossis I, Dickerson FB, Stallings CR, Origoni AE, Sullens A, et al. Development of a nucleocapsid-based human coronavirus immunoassay and estimates of individuals exposed to coronavirus in a U.S. metropolitan population. Clin Vaccine Immunol. 2008;15(12):1805-10. https://doi.org/10.1128/CVI.00124-08.

32. Struck F, Schreiner P, Staschik E, Wochinz-Richter K, Schulz S, Soutschek E, et al. Vaccination versus infection with SARS-CoV-2: establishment of a high avidity lgG response versus incomplete avidity maturation. J Med Virol. 2021;93(12):6765-77. https://doi.org/10.1002/jmv.27270.

33. Jalkanen $P$, Kolehmainen $P$, Häkkinen HK, Huttunen $M$, Tähtinen PA, Lundberg R, et al. COVID-19 mRNA vaccine induced antibody responses against three SARS-CoV-2 variants. Nat Commun. 2021;12(1):3991. https:// doi.org/10.1038/s41467-021-24285-4.

34. Planas D, Veyer D, Baidaliuk A, Staropoli I, Guivel-Benhassine F, Rajah MM, et al. Reduced sensitivity of SARS-CoV-2 variant Delta to antibody neutralization. Nature. 2021;596(7871):276-80. https://doi.org/10.1038/s41 586-021-03777-9.

35. Wall EC, Wu M, Harvey R, Kelly G, Warchal S, Sawyer C, et al. Neutralising antibody activity against SARS-CoV-2 VOCs B.1.617.2 and B.1.351 by BNT162b2 vaccination. Lancet. 2021;397(10292):2331-3. https://doi.org/10.1 016/S0140-6736(21)01290-3.

\section{Publisher's Note}

Springer Nature remains neutral with regard to jurisdictional claims in published maps and institutional affiliations. 